\title{
Karoline Dominika Döring, Sultansbriefe
}

\section{Benoît Grévin}

\section{OpenEdition \\ Journals}

Édition électronique

URL : http://journals.openedition.org/ifha/10113

DOI : 10.4000/ifha. 10113

ISSN : 2198-8943

Éditeur

IFRA - Institut franco-allemand (sciences historiques et sociales)

\section{Référence électronique}

Benoît Grévin, « Karoline Dominika Döring, Sultansbriefe », Revue de l'IFHA [En ligne], Date de recension, mis en ligne le 19 mai 2019, consulté le 24 septembre 2020. URL : http://journals.openedition.org/ ifha/10113; DOI : https://doi.org/10.4000/ifha.10113

Ce document a été généré automatiquement le 24 septembre 2020.

(C)IFHA 


\section{Karoline Dominika Döring, Sultansbriefe}

\section{Benoît Grévin}

\section{RÉFÉRENCE}

Karoline Dominika Döring, Sultansbriefe. Textfassungen, Überlieferung und Einordnung, Wiesbaden : Harrassowitz Verlag, 2017, 138 p., $35 €$ 
J'ai d'autant plus le devoir de présenter dans une tribune francophone le livre de Karoline Dominika Döring Sultansbriefe que je me suis rendu coupable d'une grave négligence scientifique concernant son objet, en affirmant dans un article paru en 2013 (Grévin, « Documents diplomatiques, diffusion des savoirs rhétoriques et problèmes d'interprétation des versions latines de correspondances réelles et fictives avec les souverains d'Orient", dans D. Aigle-S. Péquignot, $L a$ correspondance entre souverains, p. 51-75) que la tradition des «lettres du sultan» dont il traite était faiblement diffusée, sur la foi d'une bibliographie datée et en toute méconnaissance de l'excellent travail préliminaire de Bettina Wagner paru sur

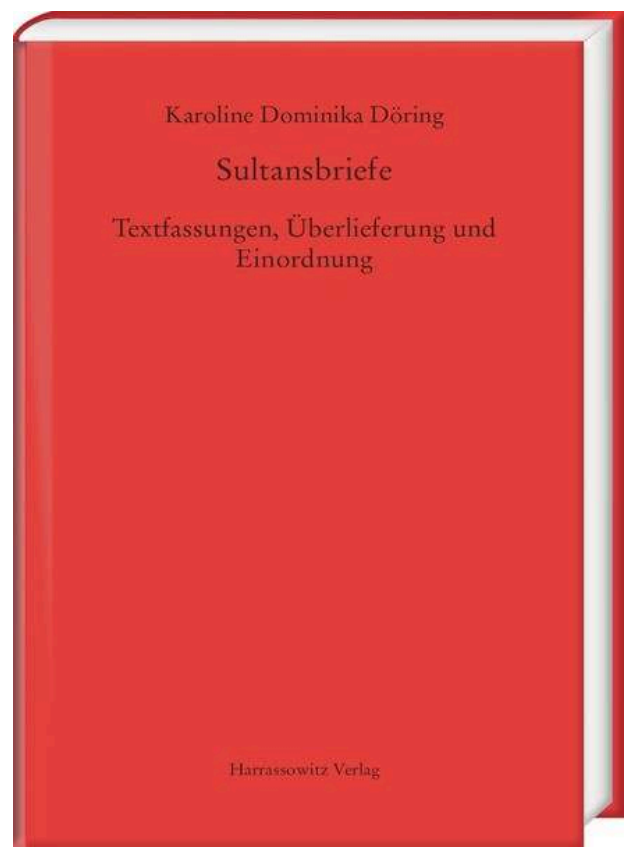
le sujet en 1999 (cf. la juste critique de K.

D. Döring p. 3). Or Sultansbriefe, centré sur la diffusion manuscrite et imprimée des «Lettres du sultan » du XIV ${ }^{e}$ au XVI ${ }^{e}$ siècles, démontre au contraire que ce sous-genre épistolaire des lettres fictionnelles entre souverains « orientaux » et " latins » (il s'agit souvent, non toujours, d'un couple de lettres échangés entre le sultan et le pape ou, moins souvent, un autre souverain) a bénéficié d'une très vaste diffusion dans tout l'Occident, et en particulier dans l'ère germanophone, à la charnière du Moyen Âge et de l'époque moderne.

Le livre se présente en effet comme un catalogue de pas moins de 158 manuscrits remontant parfois au XIV siècle, ainsi que de dizaines d'imprimés du XV et du XVI ${ }^{\mathrm{e}}$ siècle contenant des versions latines ou allemandes de ces lettres, en les ventilant en fonction de leurs deux traditions principales (Epistola soldani, Epistola Morbosani), et de trois traditions secondaires (proposition d'alliance matrimoniale, lettre de défi, lettre d'invitation au tournoi à un souverain), tout en incluant également la description de lettres isolées qui peuvent être rattachées au genre global sans rentrer dans ces catégories (Anhang, Abweichende Fassungen, p. 122-126). Une courte introduction bibliographique et méthodologique, délimitant notamment les frontières du genre par rapport à des traditions connexes (lettres du Prêtre Jean), et une discussion finale sur le statut «littéraire» (ou plutôt littéraro-historique) des "Sultansbriefe» p. 90-121) encadrent ce catalogue des manuscrits, contribuant à faire du petit livre un outil incontournable pour la recherche sur la question des correspondances entre monde chrétien et non chrétien au bas Moyen Âge et à la Renaissance, et de la dynamique de construction des rapports imaginaires et réels entre la Chrétienté et l'Islam.

La réflexion sur le statut fictionnel de ces lettres et ses limites est particulièrement pertinente, car la contextualisation des lettres dans les manuscrits permet de souligner à quel point la frontière entre textes fictionnels et non fictionnels doit être relativisée : les lettres, d'abord échangées entre un sultan de Babylone et le pape, puis de plus en plus souvent entre un sultan "ottomanisé » et le pape ou un souverain, se retrouvent souvent dans des mélanges de "Turcica» qui contiennent de véritables relations 
historiques. Les pages sur le changement progressif d'autorité des lettres, en partie en liaison avec le passage du manuscrit à l'imprimé, et la cristallisation de la figure de Pie II comme modèle du pape en opposition dialectique face à l'Islam, sont également passionnantes. Ces lettres sont, comme le dit K. D. Döring, ce que le lecteur médiéval et renaissant, préparé par l'existence de ce sous-genre, en fait. Par sa structure et sa teneur, le livre s'apparente également à un Hilfsmittel des MGH, car il permet d'étudier les regroupements textuels au fil des manuscrits et de faire apparaitre des évolutions significatives. Une grande partie des manuscrits de l'Epistola soldani (version la plus ancienne, surgie au début du XIV siècle) relie les lettres à une littérature de controverse théologique, ou de rhétorique liée à l'ars dictaminis, tandis que l'environnement textuel des epistolae Morbosani apparait beaucoup plus humaniste. Les voies pour explorer la perception de ce sous-genre foisonnant et évolutif sont donc multiples, le rapprochement récurrent avec la Historia destructionis Troiae de Guido Colonna d'une partie de la tradition pouvant ainsi être relié à la protohistoire de la "teucrisation" des Turcs également évoquée par K. D. Döring comme l'une des innombrables questions soulevées par ces textes.

Tel qu'il se présente, ce livre qui est à la fois une «introduction à » et un catalogue de cette littérature, possède une nette dimension germanique, car pour des questions aussi bien de dimension que de stabilité scientifique, l'autrice, qui traite de l'ensemble de la documentation subsistant en latin, n'y a pas inclus d'enquête sur les versions de ces lettres existant ou pouvant exister dans d'autres langues modernes (ainsi que sur l'inclusion de lettres analogues dans les chroniques, voir ses remarques méthodologiques à ce sujet, ou de certaines traditions humanistes particulières qui dévient trop du modèle polymorphe des «Sultansbriefe » tel qu'il est ici défini). On ne peut que souhaiter à ce sujet que ce livre serve de modèle à des répertoires analogues prolongeant cette enquête dans différentes zones linguistiques de l'Europe. Peut-être le livre aurait-il par ailleurs encore gagné à présenter quelques éditions de travail effectuées à partir de manuscrits particulièrement intéressants ou représentatifs d'une ou deux des lettres discutées, mais on peut comprendre que tel n'était pas son propos.

En ce qui me concerne, je tenterai volontiers dans les années qui viennent de réparer ma faute professionnelle en approfondissant dans le futur la question des prototypes ou sources d'inspiration possibles au genre autonome des "Lettres du/au sultan = Sultansbriefe » apparu au XIV siècle, à partir de certains filons indiqués dans mon article fautif en ce qui concerne les «Sultansbriefe » cité supra (les lettres de défi de Frédéric II et du sultan de Babylone de la tradition de Pierre de la Vigne, par exemple), ou d'autres sources pertinentes présentes dans des traités d'ars dictaminis du XIII ${ }^{\mathrm{e}}$ siècle avec lesquelles des rapprochements pourraient être faits (échange entre un sultan et le roi de Castille dans l'Ars epistolaris ornata inédite de Gaufridus d'Everseley, modèles de salutationes variables d'autorités chrétiennes aux sultans d'Egypte dans le Pomerium rhetorice de Bichilino da Spello, etc...). Il ne me reste qu'à recommander la lecture attentive de Sultansbriefe à tous les chercheurs travaillant sur le vaste champ des relations - réelles ou imaginées - entre le monde musulman et l'Occident latin du XIV ${ }^{\mathrm{e}}$ au $\mathrm{XVI}^{\mathrm{e}}$ siècle, mais aussi plus généralement à la question des relations entre les champs littéraires, épistolaires et historiques au Moyen Âge et à la Renaissance. 
INDEX

Index chronologique : Moyen Âge, Période moderne

Thèmes : Sources, Histoire des idées

\section{AUTEURS}

BENOÎT GRÉVIN

CNRS-CRH (UMR 8558) 